\title{
Genome-wide association study in wheat to identify resistance against Pratylenchus thornei and Heterodera avenae
}

\author{
Sharma Shailendra ${ }^{1 *}$, Sharma Shiveta ${ }^{1}$, Kumar D. ${ }^{1,2}$, Singh V.K. ${ }^{1}$, Pundir S. ${ }^{1,2}$, \\ Chaturvedi D. ${ }^{1}$ \\ ${ }^{1}$ Department of Genetics and Plant Breeding, Chaudhary Charan Singh University, Uttar Pradesh, India \\ ${ }^{2}$ Department of Botany, Chaudhary Charan Singh University, Uttar Pradesh, India \\ *email:Shgjus6@gmail.com
}

\begin{abstract}
Bread wheat (Triticum aestivum L.), is one of the most important staple food crops of the world. Production of wheat can be limited by various biotic factors, including plantparasitic nematodes (PPNs) which are also known as the 'hidden enemy' of crops as they pose a serious threat to global food security. PPNs cause losses of $\sim 12.6 \%$ among different crop plants, representing an annual monetary loss of 216 billion US\$. The most damaging PPNs are the root-knot nematodes (RKNs), cereal cyst nematodes (CCNs) and root lesion nematodes (RLNs). Identification of the novel resistance sources is of high importance for applied breeding. In the present study, 143 and 323 wheat genotypes were screened against RLN (Pratylenchus thornei) and CCN (Heterodera avenae) respectively. GWAS was conducted to find out the significant marker-trait associations (MTAs) underlying $P$. thornei resistance. A total of 25 MTAs were obtained which were further explored to find out putative candidate genes. Ten highly resistant and 10 highly susceptible genotypes were selected from these 143 wheat genotypes and further validated to confirm resistance for $P$. thornei. GWAS analysis to find MTAs for $H$. avenae resistance in 323 wheat genotypes is underway.
\end{abstract}

